\title{
The Larval Stage of Echinococcus Granulosus
}

Mohsen Sokouti ${ }^{1^{*}}$

1. Professor of Surgery, Department of Surgery, Tabriz University of Medical Sciences, Tabriz, Iran

Throughout the life cycle of Echinococcus granulosus, eggs of the parasite cannot mature into adult worms without first passing through the larval stage. Regarding the fact that this stage cannot take place in the definitive host, the eggs must look for an intermediate host, such as humans which are considered accidental intermediate hosts, in order to undergo their vital metamorphosis. In the upper gastrointestinal tract of the intermediate host, including humans (but not that of definitive host), the outer chitinous shells of the hexacanth embryos become lysed, enabling the embryos to penetrate the mucosa of the duodenum and upper jejunum to enter mesentric venule and be carried in the portal stream to the liver. Theoretically, a few of the embryos can enter the lymphatics of the intestinal wall and bypassing the liver through the cisterna chyli (1-3).

It is believed that the larger amount of deoxycholic acid in the bile of herbivores and humans conjugated principally with glycine is responsible for lysis of the larva's protective center cuticle. On the other hand bile salts of carnivores such as dog are relatively poor in deoxy cholic acid which is linked with urine and have no effect on the cuticle of the larvae, which remain in the bowel lumen and developing into adult worms. Thus unlike what is mostly believed, humans do not serve as definitive hosts for the parasite; yet they carry only the larval forms which later penetrate into the villi of small bowel and form hydatid cyst in any organ of body (4-6).

\section{References}

1. Golzari SE, Sokouti M, Ghaffari A, Bazzazi AM, Ghabili K. Ultrasonography in diagnosis of pulmonary hydatid cysts. Lancet Infect Dis. 2013;13(4):294.

2. Golzari SE, Sokouti M, Bazzazi AM, Khanli HM, Ghabili K. Serodiagnostic tests in musculoskeletal hydatid disease. Spine (Phila Pa 1976). doi: 10.1097/BRS.0b013e3182a0038c).

3. Sokouti M, Golzari SE, Tizro P, Khanli HM, Ghabili K. Genitourinary hydatid disease. Int Urol Nephrol. 2013;45(3):757-8. doi: 10.1007/s11255-013-0440-0.

4. Sokouti M, Golzari SE, Kayhan S, Sabermarouf B. Recurrence Following Pulmonary Hydatid Disease Surgery. World J Surg. doi: 10.1007/s00268-013-2141-3.

\section{Corresponding author:}

Mohsen Sokouti, MD

Professor of Surgery, Department of Surgery, Tabriz University of Medical Sciences, Tabriz, Iran

E-mail: sokouti.mohsen@yahoo.com

Receive date: 2014-04-01| Accept date: 2014-04-29 | Publish date: 2014-05-08

DOI: 10.7575/aiac.abcmed.15.03.01.03

\section{Al}


5. Sokouti M, Golzari SE, Ghabili K, Bazzazi AM. Pathogenesis of cerebral hydatid disease. Neurosciences (Riyadh). 2013;18(4):393-4.

6. Golzari SE, Sokouti M. Pericyst: the outermost layer of hydatid cyst. World J Gastroenterol. 2014 7;20(5):1377-8. doi: $10.3748 /$ wjg.v20.i5.1377. 